\title{
Power Quality in Wind Power Systems
}

\author{
Z. Leonowicz \\ Department of Electrical Engineering \\ Wroclaw University of Technology \\ Wyb. Wyspianskiego 27 - Wroclaw, 50370 Wroclaw (Poland) \\ Phone/Fax number:+0048 71 3202626/+0048 71 3202006,e-mail: leonowicz@ieee.org
}

\begin{abstract}
Wind power generation systems influence power quality in a specific way making important the need for detailed and accurate evaluation of disturbances caused by wind generators. Wind power system can cause sub- and interharmonic components to appear in the spectrum of voltages and currents.

The paper shows different aspects of spectrum estimation in power systems, including the doubly fed induction generator (DFIG) connected to low voltage distribution grid. Harmonic distortions caused by wind generator under various operational conditions regarding wind speed, active and reactive power are shown and discussed. Advanced spectral methods (like ESPRIT) are applied to overcome the drawbacks of Fourierbased techniques.
\end{abstract}

\section{Key words}

power quality, subharmonics, interharmonics, parametric spectral estimation.

\section{Introduction}

Wind generation is one of the most mature and cost effective resources among different renewable energy technologies [1]. Further intensification of wind farms proliferation into electric systems is supported by ecologists and governments. The long-term target of European Union is to increase the share of renewable energy resources to $22 \%$ [2].

The current policies imply the use of wind turbines with good grid compatibility and integrated measures for grid reinforcement [4].

There exist various design concepts of wind generators which allow fulfilment of regulatory requirements [4], concerning grid stability. Examples are conversion systems with synchronous generator, converter-inverter units, short-circuited rotor asynchronous machines also with converter-inverter units and asynchronous machines with static compensators [8].

One of the most efficient designs is the doubly fed induction generator DFIG [10], which allows the regulation of reactive power and the adjustment of angular velocity to maximize the output power efficiency. These generators can also support the system during voltage sags.
However, the drawbacks of converter-based systems are harmonic distortions injected into the system [11]. The paper is devoted to the evaluation of harmonic distortion in various operation conditions of the wind generation units.

Parametric spectral estimation ESPRIT algorithm [8] is applied to extract information on harmonics parameters. It is assumed that those advanced methods could give more detailed information, then traditional FFT.

The signals under investigation were obtained from the laboratory model of DFIG which allowed quite free regulation of active and reactive power and angular velocity of the shaft.

The interest was focused on the harmonics generated by DFIG. Due to restrictions imposed by utilities on wind park operators it is difficult to run such research activity on real object within widespread range of parameters changes in acceptably short time.

In the first part, the physical, grid connected model consisting of induction generator, direct converter, DC machine and control unit is introduced. In the second part of the paper, the ESPRIT algorithms are presented. The measurement procedure and assessment of grid parameters follow. Current measurements and harmonics estimation results are finally presented and discussed.

\section{Measuring Inter-Harmonics}

Inter-Harmonics as defined in IEC-1000-2-1 [5] are spectral components which appear "between the harmonics of the power frequency voltage and current...". Also: "Frequencies can be observed which are not integer multiples of the fundamental. They can appear as discrete frequencies or as a wide-band spectrum".

However, as pointed out in [5], the currently employed methods, based on Fourier transform, are not always suitable for the estimation of inter-harmonics.

In real-world problems, the electric signal under investigation is seldom perfectly periodic and it is impossible to obtain the recordings of infinite length which constitutes an important violation of basic requirements for accurate Fourier analysis. Windowing of the signal assumes that the signal is self-repeating in time. In reality, the spectral analysis is performed over consecutive time windows, yielding a time-varying timefrequency representation [5]. 
Reliable measurement of inter-harmonics using Fourierbased methods demands the exact adjustment of the window length, taking into account the frequency of the fundamental components and common divider of all interharmonic components. This results in a very long window. For non-stationary signals, commonly encountered in wind power systems, additional problems appear. Firstly, the time-varying spectral components can appear as additional inter-harmonics or the results can be inaccurate. Such spurious components are not real spectral components but only an inherent by-product of the Fourier analysis.

\section{Sub-Harmonics}

Sub-harmonics are inter-harmonics with frequency lower than fundamental frequency [7]. Sub-harmonics can be generated by some of contemporary loads: among others cycloconverters, arc furnaces, motors driving cyclic loads, rectifiers and wind generators (as a consequence of tower-to blade interaction causing the modulation of the generator torque) [6]. Consequences of voltage subharmonics, even for very low amplitudes are important. Even a single sub-harmonic tone is able to cause light flicker. According to recent studies, all interharmonic components from 15 to $85 \mathrm{~Hz}$ can cause light fluctuations [6]. Furthermore, sub-harmonics can cause asymmetrization and core saturation of transformers, thermal aging of induction motors and perturbation of generators function manifesting as sub-synchronous torques. In another study, measurements of of the r.m.s. voltage magnitude of the sub-harmonic group and subgroup, defined as grouping of the output bins of an IEC harmonic measurement instrument below $50 \mathrm{~Hz}$, were investigated in a low-voltage distribution system. The results showed considerably higher magnitude of these components than expected. [7]

Therefore, it is important to look for reliable methods of spectral analysis which can provide accurate results for short data records, thus avoiding the influence of time variation of spectral parameters on the accuracy of obtained results.

\section{ESPRIT Algorithm}

ESPRIT algorithm [8] belongs to the subspace parametric spectrum estimation methods. It allows determining the parameters of harmonic components with high accuracy.

Furthermore, the results of spectral estimation do not depend, to large extent, on the length of the analysis window. Extensive investigations shown in [13] confirm the suitability of this algorithm to provide reliable estimates. Parametric algorithm show very high accuracy in frequency estimation independently of the window length (for window lengths from 100 to 250 samples) and also relatively high accuracy in amplitude estimation, without the de-synchronization effects. Furthermore, the problem of "masking" of harmonics with low amplitude by high-amplitude components is considerably less harmful than for Fourier-based techniques.

ESPRIT algorithm is based on eigenvector decomposition of the autocorrelation matrix of the signal where two subspaces (signal and noise subspaces) are defined. The rotational invariance between both subspaces allows extracting of the parameters of spectral components present within the investigated waveform.

The assumed signal model is the following:

$$
y[n]=\sum_{k=1}^{M} A_{k} e^{\left(j \omega_{k} n\right)}+w[n]
$$

where $w[n]$ represents additive noise. The eigenvectors $\mathbf{U}$ of the autocorrelation matrix of the signal define two subspaces (signal and noise subspaces) by using two selector matrices $\boldsymbol{\Gamma}_{1}$ and $\boldsymbol{\Gamma}_{2}$.

$$
\mathbf{S}_{1}=\boldsymbol{\Gamma}_{1} \mathbf{U} \quad \mathbf{S}_{2}=\boldsymbol{\Gamma}_{2} \mathbf{U}
$$

The rotational invariance between both subspaces leads to the equation:

$$
\begin{gathered}
\mathbf{S}_{1}=\boldsymbol{\Phi} \mathbf{S}_{2} \\
\text { where: } \boldsymbol{\Phi}=\left[\begin{array}{cccc}
\mathrm{e}^{\mathrm{j} \omega_{1}} & 0 & \cdots & 0 \\
0 & \mathrm{e}^{\mathrm{j} \omega_{2}} & \cdots & 0 \\
\vdots & \vdots & \ddots & \vdots \\
0 & 0 & \cdots & \mathrm{e}^{\mathrm{j} \omega_{\mathrm{M}}}
\end{array}\right]
\end{gathered}
$$

The matrix $\boldsymbol{\Phi}$ contains all information about $M$ components' frequencies. Additionally, the TLS (total least-squares) approach (applied for solving of (3) for $\boldsymbol{\Phi}$ ) assumes that both estimated matrices $\mathbf{S}$ can contain errors and finds the matrix $\boldsymbol{\Phi}$ as minimization of the Frobenius norm of the error matrix.

\section{Model of wind generator}

Simplified model of DFIG is shown in Fig.1 [12]. The rotor of induction machine is connected to the grid with a back-to-back voltage source converter which controls the excitation system. This most significant feature enables sub synchronous and super synchronous operation speeds in generator mode and adjustable reactive power generation.

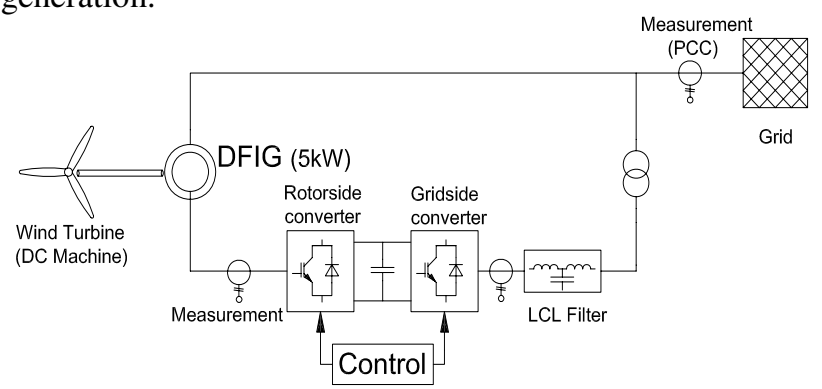

Fig. 1. Doubly fed induction generator

The wind turbine was modeled with a separately excited $30 \mathrm{~kW}$ DC machine. Torque and angular velocity could be set in a controlled manner in accordance to speedtorque characteristic of a real three blade rotor adapted for steady state operation of the $5 \mathrm{~kW}$ DFIG model. The generator in Fig. 1 is a $5 \mathrm{~kW}$, two pole pairs slip ring induction machine with synchronous speed of $1500 \mathrm{rpm}$. A LCL filter was installed behind the grid side converter to minimize harmonic distortion introduced in the grid. 
The filter components parameters were settled with accordance to grid and transformer inductances and converter characteristic.

The Y/y 230/100 V not grounded transformer was used to match the converter voltage level with the low voltage grid.

\section{Results of Analysis}

During the sub- and super-synchronous mode measurements were done at different points of the DFIG system.

The measurements were carried out during steady state operation (without transients). The power quality recorder used enabled the capture of currents and voltages with sampling frequency of $10 \mathrm{kHz}$.

\section{A. Sub-synchronous Mode}

The measurements in sub synchronous mode correspond to $1401 \mathrm{rpm}$ of the rotor. The simulation parameters were:

$\begin{array}{ll}\text { Active Power at Stator } & -1106 \mathrm{~W} \\ \text { Reactive Power at Stator } & -200 \mathrm{var} \\ \text { Intermediate Circuit Voltage } & 200 \mathrm{~V} \\ \text { Reactive Power at Rotor } & 0 \mathrm{var}\end{array}$

The mean active power value introduced into the grid at point of common coupling (pcc) was significantly smaller, about $710 \mathrm{~W}$. The total losses included losses caused by protection devices, converter, transformer and LCL filter. The active power introduced in the rotor circuit of the induction machine was about $287 \mathrm{~W}$.

Typically, the power factor at pcc during the operation of a wind generator should be equal to one. Due to unsymmetrical conditions the highest power factor achieved was about 0.94 .

Fig. 2 shows the phase current in one phase supplied into the grid, sampled with the frequency of $10 \mathrm{kHz}$.

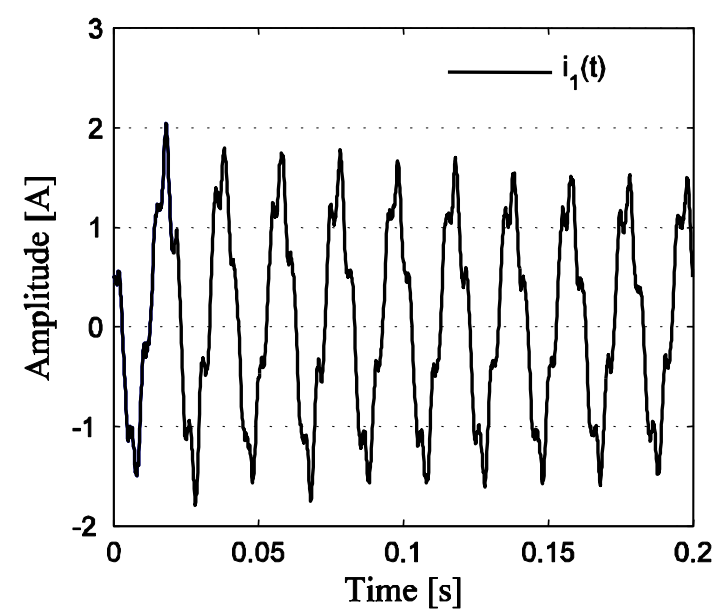

Fig. 2. Measured current in phase one in subsynchronous mode.

Spectral components were obtained using a windowed signal with the length adapted to ten periods of fundamental $50 \mathrm{~Hz}$ component.

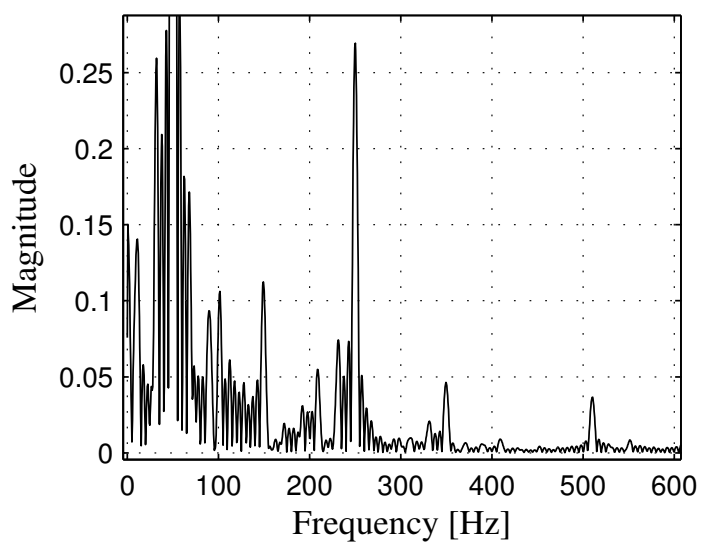

Fig. 3. Fourier spectrum of the current in subsynchronous mode.

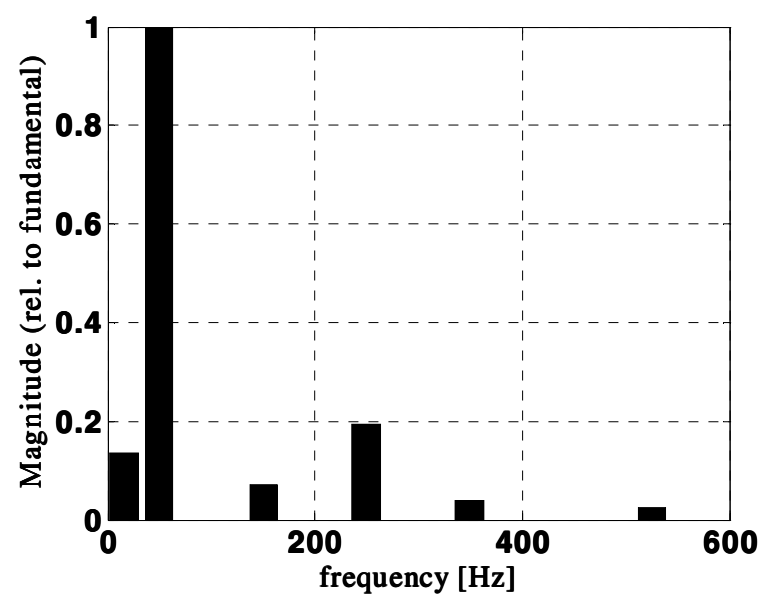

Fig. 4. Spectral components estimated using the ESPRIT algorithm in sub-synchronous mode..

TABLE I. - Spectral components estimated using the ESPRIT algorithm in sub-synchronous mode

\begin{tabular}{|c|c|}
\hline Frequency [Hz] & Amplitude [A] \\
\hline 16.20 & 0.21 \\
\hline 49.98 & 1.56 \\
\hline 150.64 & 0.11 \\
\hline 249.49 & 0.30 \\
\hline 349.26 & 0.06 \\
\hline 525.41 & 0.04 \\
\hline
\end{tabular}

ESPRIT algorithm allows detecting one subharmonic component, impossible to detect using the Fourier spectrum (Fig. 3), because of the lack of synchronization with fundamental component, which causes an important leakage effect. Parametric spectral estimation does not suffer from many shortcomings, typical for Fourier-based techniques. Moreover, in addition to third, fifth and seventh harmonic it shows the presence of one interharmonic component, clearly visible also in the Fourier spectrum.

\section{B. Super-synchronous Mode}

The power of the wind turbine was $2.9 \mathrm{~kW}$ at $1617 \mathrm{rpm}$. Contrary to sub-synchronous mode the active power is 
fed into the gird on stator and rotor side. The simulation data are summarized below:

$\begin{array}{ll}\text { Active Power at Stator } & -2800 \mathrm{~W} \\ \text { Reactive Power at Stator } & -350 \mathrm{var} \\ \text { Intermediate Circuit Voltage } & 200 \mathrm{~V} \\ \text { Reactive Power at Rotor } & 0 \mathrm{var}\end{array}$

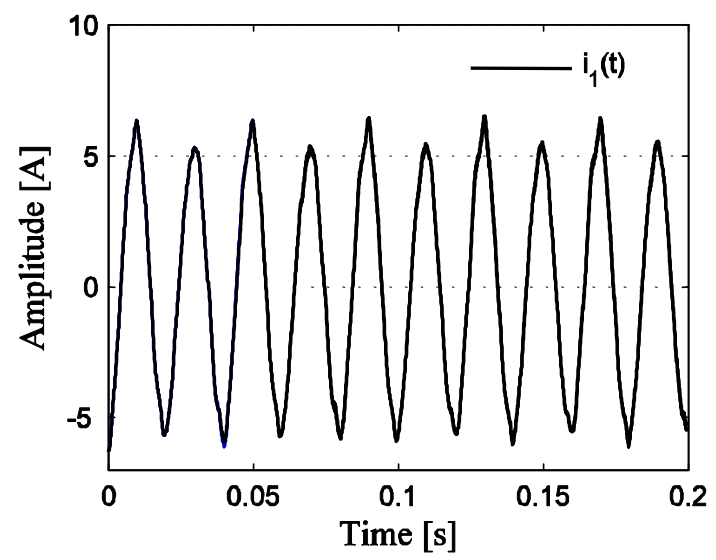

Fig. 5. Measured current in phase one in supersynchronous mode.

The power factor was kept possibly near one and was equal to 0.96 . Fig. 5 shows the measured current in phase one.

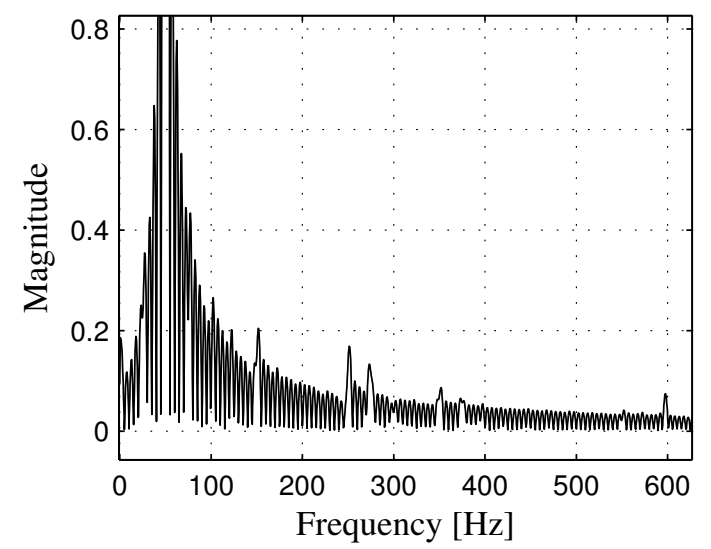

Fig. 6. Fourier spectrum of the current in supersynchronous mode.

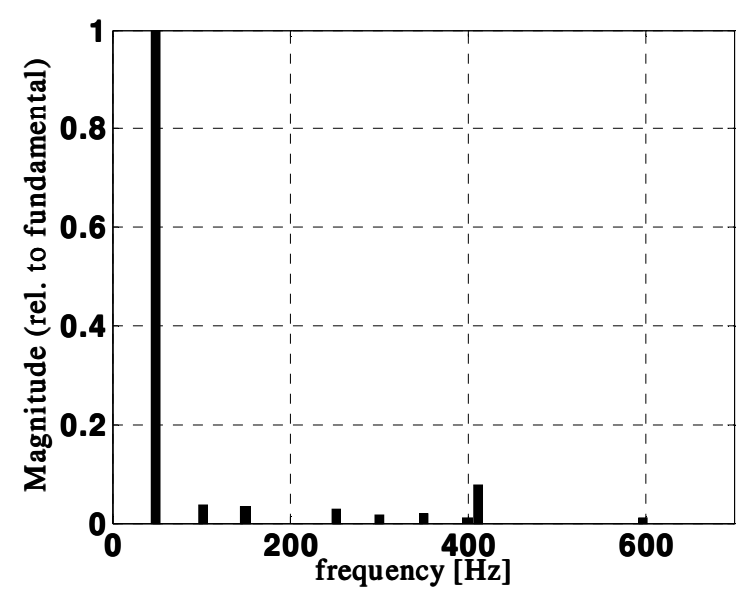

Fig. 7. Spectral components estimated using the ESPRIT algorithm in super-synchronous mode.
TABLE II. - Spectral components estimated using the ESPRIT algorithm in super-synchronous mode

\begin{tabular}{|c|c|}
\hline Frequency [Hz] & Amplitude [A] \\
\hline 49.95 & 5.52 \\
\hline 102.79 & 0.20 \\
\hline 149.99 & 0.18 \\
\hline 252.20 & 0.16 \\
\hline 300.10 & 0.09 \\
\hline 351.42 & 0.10 \\
\hline 400.01 & 0.05 \\
\hline 412.20 & 0.43 \\
\hline 598.07 & 0.05 \\
\hline
\end{tabular}

In this case, the current spectrum contains in general less harmonic distortion than in the sub-synchronous mode, which makes the correct spectrum estimation even more difficult (due to "masking" effect which affects more the Fourier-based methods). Beside the expected odd harmonic components, also even harmonic components are present in the current spectrum, as well as interharmonic component. Detection and resolution capabilities of ESPRIT method clearly outperform the Fourier-based classical method.

\section{Conclusions}

Currents fed into the grid by the investigated DFIG system have a considerable harmonic content. Not only characteristic harmonics but also uncharacteristic harmonics interharmonics and subharmonics were observed. In the current practice, interharmonics are often not taken into account during power quality compatibility tests of wind generator systems and the analysis results revealed significant levels of this type of distortion.

Monitoring and accurate estimation of power quality parameters is a crucial issue in guaranteeing reliable and safe operation of a power system with wind generation. That is also an important prerequisite for an adequate design of harmonic mitigation systems.

Parametric spectral estimation ESPRIT method, accurately and reliably showed high content of interharmonics in the current waveforms, justifying their usefulness as a tool for spectral analysis of distorted electric signals in wind power generators.

\section{References}

[1] T. Hammons, "Status of integrating renewable electricity production in Europe into the grids", Proceedings of UPEC Conference, Cork (Ireland), 2005, p.75

[2] 2001/77/EC Directive on the promotion of electricity produced from renewable energy sources in the internal electricity market

[3] S. Heier, "Grid Integration of Wind Energy Conversion Systems", Chichester, Wiley, 2006

[4] R. Chapman, "Reliability and Quality You get What you expect" in The Evolution of Electric Power Trans-mission under Deregulation, IEEE Press, Piscataway, 2000, pp.187-195 
[5] C. Li, W. Xu, "On the Ambiguity of Defining and Measuring Inter-Harmonics, IEEE Power Engineering Review, July 2001, pp. 56-57

[6] A. Testa, R. Langella, "Power System Subharmonics", 2005. IEEE Power Engineering Society General Meeting, 12-16 June 2005, Vol. 3, pp.: $2237-2242$

[7] J. Barros, M. de Apraiz, R. I. Diego, "Measurement of Subharmonics in Power Voltages 2007 IEEE Lausanne Power Tech, 1-5 July 2007, pp. 17361740

[8] J. Arrillaga, N. R. Watson, "Power System Quality Assessment", Chichester, Wiley, 2000

[9] G. J. Wakileh "Power System harmonics. Fundamentals, Analysis and Filter Design", Berlin, Springer, 2001
[10] Y.Lei, A. Mullane, G. Lighbody, R. Yacamini, "Modeling of the Wind Turbine with a Doubly Fed Induction Generator for Grid Integration Studies", IEEE Trans. On Energy Conversion, vo. 21, No. 1, march 2006, pp. 257-264

[11] C. W. Therrien, „Discrete Random Signals and Statistical Signal Processing“, Englewood Cliffs, NJ:1992

[12] P. Janik, Z. Leonowicz, J. Rezmer, S. Chistan, D. Schulz: "Advanced Signal Processing Methods for Evaluation of Harmonic Distortion Caused by DFIG Wind Generator", Proceedings of the 16th Power Systems Computation Conference, PSCC 2008, Glasgow, Scotland, 14-18 July, 2008, paper No. 78.

[13] Z. Leonowicz, T. Lobos, T. Sikorski, "TimeFrequency Analysis of Complex Space-Phasor in Power Electronics", IEEE Trans. on Instrumentation and Measurement, Vol. 56, No. 6, Dec 2007, pp. 2395-2403 